\title{
EDITORIAL
}

\section{Observational vs randomized: David vs Goliath for thromboprophylaxis in critically ill patients?}

Julie Helms ${ }^{1,2,3^{*}}$ (D) Julian Bion ${ }^{4}$ and Audrey De Jong ${ }^{5,6}$

(C) 2019 Springer-Verlag GmbH Germany, part of Springer Nature

In their article in Intensive Care Medicine, Stelfox et al. [1] report the impact of a multicomponent implementation intervention on the clinical practice of pharmacological venous thromboembolism (VTE) prophylaxis, related outcomes, and healthcare utilization among patients admitted to 11 Canadian ICUs. Their intervention resulted in a $32.4 \%$ increase in the administration of low molecular weight heparin (LMWH) over unfractionated heparin (UFH) over the course of 12 months in the intervention group (five ICUs), significantly more than in the control group (15.4\%).

Thromboprophylaxis of critical care patients is a major clinical and economical issue. Critically ill patients are at higher risk of venous thromboembolism, which is associated with longer duration of mechanical ventilation, intensive care unit stay, and hospitalization [2,3]. Randomized controlled trials (RCTs) on pharmacological thromboprophylaxis [4] suggested that LMWH would be more efficacious than UFH, without increased bleeding complications or heparin-induced thrombocytopenia incidence [3]. In this setting, Stelfox et al. [1] performed a cluster-randomized trial of a multifaceted intervention to accelerate implementation of LMWH use. Quality improvement programs in intensive care seek to improve compliance by implementing best practice protocols, such as intubation procedures [5], prevention of ventilator-associated pneumonia [6], weaning from mechanical ventilation [7], and pain management [8]. The intervention designed by Stelfox et al. [1] was successful in

*Correspondence: Julie.helms@chru-strasbourg.fr

2 Service de réanimation, Hôpitaux universitaires de Strasbourg, Nouvel Hôpital Civil, 1, place de l'Hôpital, 67091 Strasbourg cedex, France

Full author information is available at the end of the article stimulating a change in practice: LMWH prescription in the control group increased from $37.9 \%$ to $53.3 \%$ which followed the pre-intervention secular trend, whereas the use of LMWH in the intervention group increased from $45.9 \%$ to $78.3 \%$ of patient days. However, contrary to the results of previous RCTs $[3,4]$, the change in practice stimulated by the intervention was not accompanied by a between-group pre- versus post-implementation difference in patient outcomes [1]: there was no significant difference in the incidence of venous thromboembolism, length of stay, mortality, or healthcare costs.

The study by Stelfox et al. [1] has several major strengths. First, the authors tailored to each ICU a multicomponent intervention to encourage the use of $\mathrm{LMWH}$ for VTE prophylaxis, based on available local resources and involved usual multidisciplinary staff (nurses, physicians, pharmacists). Then, a careful examination of exclusion criteria and patient characteristics confirms that the authors did include an all-round critical care population, as consecutive medical, surgical, and cardiovascular surgical patients admitted to ICUs were included in the study unless they presented with a primary bleeding disorder, neurological disorder, or injury. As a consequence, all patients potentially eligible for pharmacological prophylaxis-12,342 patients from a population of 17,242 patients $(72 \%)$ admitted to the ICUs-were included in the study. Further, the size of the sample studied is a major determinant of the risk of reporting false negative findings. For example, to detect a difference between two groups of $1 \%$ (from $3 \%$ to $2 \%$ ), with an alpha risk of $5 \%$, sample sizes of 6000 and 10,000 patients would respectively allow a $70 \%$ and $89 \%$ power of the study, as shown in Fig. 1a. In the study by Stelfox et al. [1], the risk of finding no difference when a true difference did exist was

\section{Springer}


very low. The inclusion of 12,342 patients allowed a type II error of $6 \%$ for detecting a difference of $1 \%$ (from $3 \%$ to $2 \%$ rate of VTE) between the two groups, corresponding to an elevated power of $94 \%$ (Fig. 1b) [1]. This sample size was also enough to conclude an equivalence [9] between groups with an equivalence limit of $1 \%$, with a power of more than $90 \%$. Moreover, the difference-in-difference statistical analysis using interrupted time series analysis with segmented linear regression models was the method of choice to assess the effects of a multicomponent intervention over time [10].

We can, however, make some observations. First, the rate of VTE is lower than that previously reported in the literature, with $1.4-1.9 \%$ of patients experiencing deep vein thrombosis and $1.2-1.3 \%$ pulmonary embolism before implementation of study intervention, compared to as high as $10 \%$ for deep vein thrombosis and $2-4 \%$ for pulmonary embolism in previous reports $[2,3]$. The headroom for further improvement was therefore limited. Then, despite the wide inclusion criteria, few exclusion criteria and a multicenter design (11 ICUs in nine hospitals in two cities), both ICU and hospital mortality rates $(8.3-10.2 \%$ and $13.0-13.3 \%$, respectively) and ICU and hospital lengths of stay (3.8-3.9 days and 11.5-12.3 days, respectively) of the included patients were low, consistent with APACHE II and SOFA scores, despite including a preponderance of non-operative patients (53-55\%). These points may limit generalizability of the results: more seriously ill patients with a higher VTE risk might show greater benefit from this intervention.

Finally, Stelfox et al. [1] illustrate the well-known discrepancy between trials demonstrating efficacy (the intervention works in clinical trials under optimum conditions) and studies assessing effectiveness (the intervention works in the real world) [11-13]. Failure
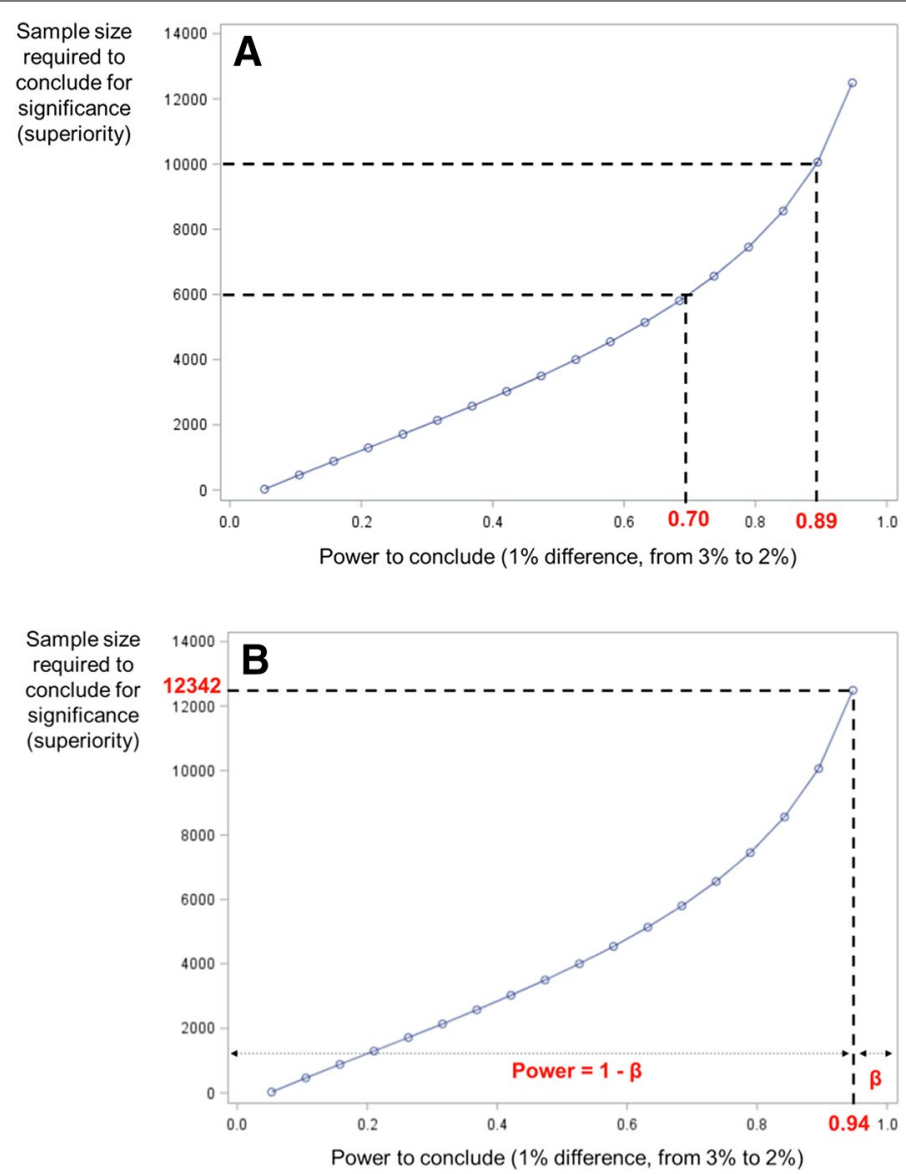

Fig. 1 Required sample size to detect a difference of 1\% between groups in rate of VTE (from 3\% to 2\%) according to the level of power (type II error). To detect a difference between two groups of 1\% (from 3\% to 2\%), with an alpha risk of 5\%, sample sizes of 6000 and 10,000 patients would respectively allow a 70\% and 89\% power of the study, as shown in a. In the study by Stelfox et al. [1], the risk of reporting that there was no difference when a true difference existed was very low. The inclusion of 12,342 patients allowed a type II error of $6 \%$ for detecting a difference of $1 \%$ (from $3 \%$ to $2 \%$ rate of VTE) between the two groups, corresponding to an elevated power of $94 \%$ (b) 
to demonstrate efficacy is likely a consequence of performing it in an all-round critical care population with diverse characteristics, rather than in highly selected patients under strictly controlled conditions and complete protocol adherence as highlighted by the authors. Glasgow et al. [12] suggested that negative effectiveness trials may result from a lack of proper implementation or weak acceptance or adherence by participants. In the present study, patients in the intervention group received LMWH on $78.3 \%$ of days, which was lower than in previous RCTs, but consistent with the pragmatic study design.

Whether there is a substantial benefit in implementing thromboprophylaxis by LMWH over UFH in critical care patients remains uncertain, considering that UFH has some advantages over LMWH (shorter halflife, easy to modify dose, anticoagulation reversion) in patients at higher risk of developing bleeding complications, as well as avoiding the contraindications to LMWH such as kidney injury. We would also note that complex behavior change interventions in prescribing could be replaced or supplemented by the forcing functions of intelligent electronic prescribing systems with decision support based on large data sets [14]. Large well-conducted randomized controlled effectiveness studies and observational studies provide important insights into the link between practice and outcomes [15].

\begin{abstract}
Author details
${ }^{1}$ Faculté de Médecine, Université de Strasbourg (UNISTRA), Strasbourg, France. 2 Service de réanimation, Hôpitaux universitaires de Strasbourg, Nouvel Hôpital Civil, 1, place de l'Hôpital, 67091 Strasbourg cedex, France. ${ }^{3}$ ImmunoRhumatologie Moléculaire, INSERM UMR_S1109, LabEx TRANSPLANTEX, Centre de Recherche d'Immunologie et d'Hématologie, Faculté de Médecine, Fédération Hospitalo-Universitaire (FHU) OMICARE, Fédération de Médecine Translationnelle de Strasbourg (FMTS), Université de Strasbourg, Strasbourg, France. ${ }^{4}$ Department of Intensive Care Medicine, University of Birmingham, Edgbaston, Birmingham B15 2TJ, UK. ${ }^{5}$ PhyMedExp, University of Montpellier, INSERM U1046, CNRS UMR 9214, 34295 Montpellier Cedex 5, France. ${ }^{6}$ Anesthesia and Critical Care Department B, Saint Eloi Teaching Hospital, Centre Hospitalier Universitaire Montpellier, 34295 Montpellier Cedex 5, France.
\end{abstract}

\section{Compliance with ethical standards}

\section{Conflicts of interest}

The authors have no conflict of interest to declare.

\section{Ethical approval}

An approval by an ethics committee was not applicable.
Received: 18 January 2019 Accepted: 19 January 2019

Published online: 28 January 2019

\section{References}

1. Stelfox HT, Brundin-Mather R, Soo A, Parsons Leigh J, Niven DJ, Fiest KM, Doig CJ, Zuege DJ, Kushner B, Clement F, Straus SE, Cook DJ, Bagshaw SM, Sauro KM (2019) A multicentre controlled pre-post trial of an implementation science intervention to improve venous thromboembolism prophylaxis in critically ill patients. Intensive Care Med. https://doi. org/10.1007/s00134-019-05532-1

2. Cook D, Crowther M, Meade M, Rabbat C, Griffith L, Schiff D, Geerts W, Guyatt G (2005) Deep venous thrombosis in medical-surgical critically ill patients: prevalence, incidence, and risk factors. Crit Care Med 33:1565-1571

3. Alhazzani W, Lim W, Jaeschke RZ, Murad MH, Cade J, Cook DJ (2013) Heparin thromboprophylaxis in medical-surgical critically ill patients: a systematic review and meta-analysis of randomized trials. Crit Care Med 41:2088-2098

4. Cook D, Meade M, Guyatt G, Walter S, Heels-Ansdell D, Warkentin TE, Zytaruk N, Crowther M, Geerts W, Cooper DJ, Vallance S, Qushmaq I, Rocha M, Berwanger O, Vlahakis NE (2011) Dalteparin versus unfractionated heparin in critically ill patients. N Engl J Med 364:1305-1314

5. De Jong A, Clavieras N, Conseil M, Coisel Y, Moury PH, Pouzeratte Y, Cisse M, Belafia F, Jung B, Chanques G, Molinari N, Jaber S (2014) Implementation of a combo videolaryngoscope for intubation in critically ill patients: a before-after comparative study. Intensive Care Med 39:2144-2152

6. Landelle C, Nocquet Boyer V, Abbas M, Genevois E, Abidi N, Naimo S, Raulais R, Bouchoud L, Boroli F, Terrisse H, Bosson JL, Harbarth S, Pugin J (2018) Impact of a multifaceted prevention program on ventilator-associated pneumonia including selective oropharyngeal decontamination. Intensive Care Med 44:1777-1786

7. Zhu B, Li Z, Jiang L, Du B, Jiang Q, Wang M, Lou R, Xi X (2015) Effect of a quality improvement program on weaning from mechanical ventilation: a cluster randomized trial. Intensive Care Med 41:1781-1790

8. de Jong A, Molinari N, de Lattre S, Gniadek C, Carr J, Conseil M, Susbielles MP, Jung B, Jaber S, Chanques G (2013) Decreasing severe pain and serious adverse events while moving intensive care unit patients: a prospective interventional study (the NURSE-DO project). Crit Care 17:R74

9. Timsit JF, de Kraker MEA, Sommer H, Weiss E, Bettiol E, Wolkewitz M, Nikolakopoulos S, Wilson D, Harbarth S (2017) Appropriate endpoints for evaluation of new antibiotic therapies for severe infections: a perspective from COMBACTE's STAT-Net. Intensive Care Med 43:1002-1012

10. Wagner AK, Soumerai SB, Zhang F, Ross-Degnan D (2002) Segmented regression analysis of interrupted time series studies in medication use research. J Clin Pharm Ther 27:299-309

11. Pagoto SL, Lemon SC (2013) Efficacy vs effectiveness. JAMA Intern Med 173:1262-1263

12. Glasgow RE, Lichtenstein E, Marcus AC (2003) Why don't we see more translation of health promotion research to practice? Rethinking the efficacy-to-effectiveness transition. Am J Public Health 93:1261-1267

13. Flay BR (1986) Efficacy and effectiveness trials (and other phases of research) in the development of health promotion programs. Prev Med 15:451-474

14. Bailly S, Meyfroidt G, Timsit JF (2018) What's new in ICU in 2050: big data and machine learning. Intensive Care Med 44:1524-1527

15. Concato J, Shah N, Horwitz RI (2000) Randomized, controlled trials, observational studies, and the hierarchy of research designs. N Engl J Med 342:1887-1892

\section{Publisher's Note}

Springer Nature remains neutral with regard to jurisdictional claims in published maps and institutional affiliations. 